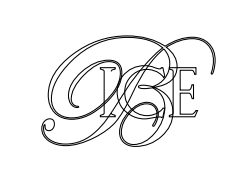

Representación Permanente de España ante los Organismos Internacionales en Ginebra*

\title{
ENTRADA EN VIGOR DEL ACUERDO DE FACILITACIÓN DEL COMERCIO
}

El 22 de febrero de 2017 entró en vigor el Acuerdo sobre la Facilitación del Comercio (AFC) de la Organización Mundial de Comercio, acuerdo que simplifica los procedimientos aduaneros de los miembros de la institución. En este artículo se hace un análisis de los plazos de aplicación del acuerdo que están asumiendo socios comerciales de especial relevancia para las empresas españolas.

Palabras clave: Organización Mundial de Comercio, Acuerdo de Facilitación del Comercio, comercio exterior, exportaciones, aduanas, aranceles, ventanilla única.

Clasificación JEL: F13, F32.

\section{Introducción: los beneficios del AFC}

El Acuerdo de Facilitación del Comercio se aprobó en la Conferencia Ministerial de la OMC de Bali de diciembre de 2013. Se trata de la primera modificación de textos legales de la OMC desde sus inicios en 1995. El AFC entró en vigor el pasado 22 de febrero para 114 miembros que culminaron su proceso de ratificación. Cuatro nuevos miembros han presentado su ratificación desde entonces, entre ellos India.

A menudo se dice que el AFC es un acuerdo win-win, con beneficios sistémicos para todos los miembros de la OMC, tanto

\footnotetext{
* Este artículo ha sido elaborado por Alberto Sanz Serrano. Representante Permanente Adjunto para Asuntos Comerciales en Ginebra.

Versión de abril de 2017.
}

para exportadores como para importadores de cualquier sector. Aun así, es cierto que para muchos países su puesta en marcha supone dificultades considerables de orden administrativo y financiero.

Desde que se negociara el Acuerdo de Facilitación de Comercio han proliferado los estudios acerca de los elevados beneficios asociados al mismo en forma de reducción de los costes al comercio, menores tiempos de espera, mayor transparencia y cooperación entre las autoridades aduaneras.

La OCDE en $2014^{1}$ estimó que la reducción de costes al comercio asociada a la aplicación plena del AFC se situaba en el 14,1 por 100 en países de renta baja, del 15,1 por 100 en países de renta media baja y del $D$

1 OCDE. The WTO Trade Facilitation Agreement - Potential Impact on Trade Costs. Trade and Agriculture Directorate. February, 2014. 
Representación Permanente de España ante los Organismos Internacionales en Ginebra

12,9 por 100 en países de renta media alta. Posteriormente (OCDE, 2017) estimó que la aplicación completa del AFC incrementaría el comercio mundial un 0,6 por 100 en relación con el escenario base, siendo el impacto mayor en los países de menor nivel de renta. La OMC, por su parte, estimó en $2015^{2}$ que la aplicación del AFC añadirá medio punto porcentual al crecimiento del PIB en el período 2015-2030. El director general de la OMC ha señalado que la aplicación completa del acuerdo tiene un potencial de reducción de costes mayor que una eventual eliminación de todos los aranceles existentes en la actualidad.

\section{Flexibilidades y cláusulas de mejor esfuerzo}

El AFC es un acuerdo muy innovador. Muchos miembros lo ven como un modelo para otras negociaciones. De hecho, ya se han formulado algunas propuestas en las discusiones de otras áreas de la OMC inspiradas en la estructura y en las flexibilidades del AFC.

En primer lugar, muchas disposiciones del AFC se formulan como «mejores esfuerzos», en lugar de como obligaciones en firme. Esta es una gran diferencia respecto de otros acuerdos de la OMC, en la que las disposiciones resultan vinculantes. Así, por ejemplo, existen compromisos de mejores esfuerzos en materias tan relevantes como la implantación de la ventanilla única aduanera, el uso de internet, la coordinación de instalaciones entre aduanas contiguas o la aceptación de copias no originales. En estas cuestiones, y en muchas otras,

2 OMC. Informe sobre el Comercio Mundial 2015. Acelerar el comercio: ventajas y desafíos de la aplicación del Acuerdo sobre Facilitación del Comercio de la OMC. resultó imposible aceptar un compromiso en firme durante las negociaciones.

De hecho, si tenemos en cuenta que los doce primeros artículos (sección I del AFC) contienen cerca de 240 puntos con disposiciones sustantivas referidas a la facilitación del comercio, sólo la mitad podrían considerarse como disposiciones en firme; las demás son compromisos de mejor esfuerzo, bajo expresiones tales como «procurarán..., en la medida que sea factible..., según proceda..., se alienta...».

Aparte de estas cláusulas de best endevour, el Acuerdo de Facilitación de Comercio contiene flexibilidades de trato especial y diferenciado a favor de los países en desarrollo, reflejadas en la sección II, que les permiten ir asumiendo los compromisos del acuerdo de manera paulatina gracias a períodos transitorios que ellos mismos definen. Así, los países en desarrollo no han de asumir automáticamente todo el acuerdo desde el momento de su entrada en vigor (compromisos de categoría $A$ ), sino que pueden consignar, si lo prefieren, un plazo de aplicación (compromisos B) o incluso, en el caso de los países menos avanzados (PMA), solicitar suficiente capacitación técnica antes de asumir el compromiso en cuestión (compromisos C). Los países desarrollados, por su parte, no se pueden acoger a estas flexibilidades y han pasado a aplicar íntegramente el AFC desde su entrada en vigor el pasado 22 de febrero. Es decir, desde que se negociara el acuerdo en 2013 hasta la entrada en vigor, los países desarrollados han tenido que adaptar sus sistemas aduaneros, en caso de que no estuvieran ya adaptados $^{3}$.

\footnotetext{
3 La puesta en marcha de la Ventanilla Única Aduanera (VUE) por parte de la Agencia Tributaria en España a principios de 2016 se enmarca en este compromiso temporal asumido en el AFC.
} 
¿Significa todo esto que el acuerdo es poco ambicioso? No necesariamente. El análisis de las notificaciones de compromisos que los países en desarrollo están manifestando querer aplicar pone de manifiesto que existe una voluntad de aplicar compromisos. No tendría sentido que muchos miembros de la OMC estuvieran solicitando plazos transitorios para cumplir compromisos de mejor esfuerzo si no tuvieran, al menos, la intención de ponerlos en práctica.

Por todo ello, no resulta posible realizar un análisis de los beneficios esperables del Acuerdo sin tener en cuenta los compromisos concretos y los períodos transitorios que esté fijando cada miembro 4 .

\section{Análisis de las notificaciones hasta la fecha}

La OMC ha elaborado una base de datos ${ }^{5}$ para el seguimiento de los compromisos que notifican los miembros. Se trata de una útil herramienta para evaluar el grado de ambición y las dificultades en la aplicación de un acuerdo tan flexible como el AFC.

Las notificaciones son realizadas por países en desarrollo, en la medida en que los países desarrollados ya han de cumplir todas las disposiciones del acuerdo desde su entrada en vigor.

Del conjunto de notificaciones recibidas hasta el momento cabe destacar que el 39 por 100 se han formulado en forma de compromisos de categoría $A$, el 2 por 100 en forma de compromisos $B$ y el 1,7 por 100 en forma de

4 Un primer análisis de la notificación de compromisos a principios de 2015 puede encontrarse en BICE, n.ํ 3060: El Acuerdo sobre la Facilitación del Comercio de la OMC.

5 www.tfadatabase.org compromisos de categoría C. Ahora bien, conviene resaltar que, de entre los países que han notificado, un 56 por 100 de las disposiciones figuran sin compromiso alguno. Hay que tener en cuenta que los miembros en desarrollo de la OMC disponen de un año (hasta el 22 de febrero de 2018) para fijar los períodos transitorios aplicables a cada compromiso, si todavía no lo han realizado.

El Anexo I muestra, para algunos miembros seleccionados de la OMC, cuál es el porcentaje de disposiciones que ya han entrado en vigor (bien por tratarse de un país desarrollado o por haber asumido un compromiso A), el porcentaje de las disposiciones que tienen ya un período transitorio conocido (categoría B) y las que no tienen plazo todavía, bien por falta de notificación o por ausencia de ratificación hasta la fecha.

Del estudio de la base de datos de la OMC se desprende asimismo que algunas disposiciones resultan claramente más difíciles de asumir que otras y que, además, las disposiciones más exigentes requerirán todavía cierto tiempo hasta que sean aplicadas por todos.

Así, resulta muy significativo el escaso número de notificaciones provenientes de países menos avanzados. De los 39 PMA miembros de la OMC sólo 16 han ratificado, de los cuales 13 han realizado notificaciones de plazos. Entre los PMA que han notificado, los compromisos más difíciles de asumir, que figuran generalmente como compromisos de categoría $\mathrm{C}$, son los que tienen que ver con la ventanilla única, la implantación de sistemas de gestión de riesgo, el compromiso de publicar estadísticas con los plazos medios de levante y la implantación de facilidades para operadores autorizados. Estos cuatro compromisos fueron concebidos en el AFC como compromisos $D$ 
Representación Permanente de España ante los Organismos Internacionales en Ginebra

de mejores esfuerzos, no estrictamente vinculantes. Llama la atención que, aun así, estos países estén solicitando asistencia técnica, lo que puede estar reflejando su voluntad de cumplir con compromisos del acuerdo, incluso cuando teóricamente fueron diseñados como «voluntarios».

A continuación, la Tabla 1 muestra, de entre todos los artículos del AFC, aquellos que más o menos veces se notifican como compromiso A, para así identificar los compromisos que más fácil y más difícilmente se están asumiendo por el conjunto de los miembros.

Para valorar adecuadamente estas notificaciones, hemos procedido a añadir una primera columna a la tabla en la que se refleja si el compromiso tiene el carácter firme (F) o ha sido redactado como compromiso de mejor esfuerzo (ME). Esta calificación es necesariamente subjetiva, puesto que muchos artículos contienen subapartados en los que los compromisos bien se refuerzan, bien se limitan o bien se matizan según las circunstancias, por lo que no queda siempre claro el grado de vinculación que asume cada país al comprometerse con un artículo sin mayor precisión. No obstante, parece relevante hacer esta distinción para valorar mejor el nivel de exigencia de cada compromiso.

TABLA 1

ACUERDO DE FACILITACIÓN DE COMERCIO

DISPOSICIONES QUE MÁSY MENOS VECES SE ASUMEN CON EFECTO INMEDIATO

$\%$ DE NOTIFICACIONES COMO COMPROMISOS A

\begin{tabular}{|c|c|c|c|}
\hline F/ME & Artículo & Medidas más notificadas & $\%$ \\
\hline ME & 9 & Establecimiento de mecanismo para traslado de mercancías bajo control aduanero & 81 \\
\hline $\mathrm{F}$ & 10.5 & $\begin{array}{l}\text { No realización de inspección previa a la expedición para comprobación de código arancelario o valo- } \\
\text { ración }\end{array}$ & 73 \\
\hline $\mathrm{F}$ & 10.6 & No obligación de recurrir a agentes de aduanas & 76 \\
\hline $\mathrm{F}$ & 10.9 & Establecimiento de sistemas de tráfico de perfeccionamiento activo y pasivo & 73 \\
\hline $\mathrm{F}$ & 5.2 & Información inmediata al importador en caso de retención de las mercancías & 76 \\
\hline $\mathrm{F}$ & 10.8 & Mercancías rechazadas en frontera: obligación de devolución o permitir reexpedición & 71 \\
\hline $\mathrm{F}$ & 10.7 & Obligación de mantener procedimientos en frontera comunes y uniformes & 72 \\
\hline $\mathrm{F}$ & 11 & $\begin{array}{l}\text { Libertad para mercancías en tránsito: medidas lo menos restrictivas posible, proporcionalidad de las } \\
\text { mismas, no aplicación reglamentos técnicos }\end{array}$ & 42 \\
\hline $\mathrm{F}$ & 4 & Establecimiento de procedimientos de recurso o de revisión de decisiones aduaneras & 47 \\
\hline $\mathrm{F}$ & 6.3 & $\begin{array}{l}\text { Criterios para la imposición de sanciones aduaneras: objetividad, adecuación, transparencia, proporcio- } \\
\text { nalidad, no conflictos de interés... }\end{array}$ & 56 \\
\hline F/ME & Artículo & Medidas menos notificadas & $\%$ \\
\hline ME & 10.4 & Establecimiento de ventanilla única para operaciones aduaneras & 20 \\
\hline $\mathrm{F}$ & 7.7 & Establecimiento de un régimen simplificado para operadores autorizados & 27 \\
\hline ME & 7.6 & Publicación de estadísticas sobre plazos medios de levante & 35 \\
\hline ME & 3 & $\begin{array}{l}\text { Obligación de emisión de unas resoluciones anticipadas al menos sobre clasificación arancelaria y } \\
\text { origen de la mercancía }\end{array}$ & 31 \\
\hline ME & 5.3 & Dar oportunidad a una segunda prueba en controles sobre alimentos y bebidas & 40 \\
\hline ME & 1.3 & Establecimiento de puntos de servicios de información para los operadores & 36 \\
\hline ME & 8 & Cooperación entre los diferentes tipos de organismos que intervienen en la frontera & 35 \\
\hline ME & 7.4 & Establecimiento de un sistema de gestión de riesgo para el control aduanero & 40 \\
\hline ME & 1.2 & Información aduanera disponible en Internet & 38 \\
\hline $\mathrm{F}$ & 7.5 & Prohibición de realizar auditorías con carácter posterior al despacho de aduana & 44 \\
\hline
\end{tabular}

Fuente: elaboración propia a partir de www.tfadatabase.org 


\section{Notificaciones relevantes para España}

Centremos ahora el análisis en las notificaciones de los socios comerciales más relevantes para España.

Hay que tener en cuenta que una parte sustancial de nuestros flujos comerciales se realiza con países de la UE (con los que mantenemos sistemas aduaneros comunes frente a terceros) y con países de la EFTA (con los que compartimos ciertos procedimientos aduaneros más exigentes que los del AFC). También hay que tener en cuenta que algunos países de la OMC cuentan ya con sistemas aduaneros plenamente adaptados y que han podido asumir todos los compromisos del AFC desde el mismo momento de entrada en vigor. Es el caso de economías desarrolladas como Estados Unidos, Australia, Canadá, Japón, y de otras que han notificado compromisos completos como México, Corea, Taiwán o Israel.

Así pues, los países que todavía no están plenamente adaptados al AFC a fecha de hoy representan una parte reducida de nuestra exportación, aproximadamente el 15 por 100 de la exportación de mercancías. De entre ellos, los más relevantes según el volumen de exportaciones españolas en 2016 serían Marruecos, Turquía, China, Arabia, Brasil, Chile, India, Argentina, Egipto y Emiratos Árabes Unidos (EAU).

La Tabla 2 muestra un análisis de los artículos que estos socios comerciales todavía no han notificado como compromiso A. Para cada miembro en cuestión se indica si existe algún plazo ya fijado o si el acuerdo todavía no ha sido ratificado, aun existiendo notificación. Al igual que hiciéramos más arriba, reflejaremos en una columna si los respectivos compromisos pueden ser considerados como en firme (F) o más bien como de mejor esfuerzo (ME).
En los compromisos reflejados en la Tabla 2 se concentra el mayor impacto potencial de mejora para las exportaciones españolas. Aunque la tabla resulte por sí misma muy ilustrativa, merece la pena destacar algunas cuestiones:

- Varios de nuestros principales socios comerciales (Marruecos, Argentina, Arabia Saudí, EAU) todavía no han notificado un plazo para la adopción de una ventanilla única, incluso cuando el AFC sólo establece un compromiso en forma de mejor esfuerzo: los miembros procurarán mantener o establecer una ventanilla única. Brasil, por su parte, ha realizado un esfuerzo considerable en estos últimos años, que le ha permitido asumir el compromiso como categoría A, sin plazos transitorios.

- La aplicación completa de sistemas simplificados para operadores autorizados (operadores económicos autorizados, como se denominan en las aduanas europeas) sigue siendo complicada (Chile, EAU, Brasil) para varios de nuestros principales socios. Recordemos que el acuerdo establece en este compromiso una obligación vinculante, si bien no se dice nada sobre una figura muy útil como es el acuerdo de reconocimiento mutuo de operadores autorizados ${ }^{6}$.

- Por último, llama la atención las dificultades en Marruecos y Turquía en relación con procedimientos especiales para productos perecederos. Con ambos mercados se mantiene un importante flujo de exportación e importación de productos agroalimentarios potencialmente beneficiarios de estas disposiciones.

6 La Unión Europea ya cuenta con acuerdos de reconocimiento mutuo de operadores económicos autorizados con EE UU, Suiza, Japón y China. 
TABLA 2

ACUERDO DE FACILITACIÓN DEL COMERCIO DE LA OMC ARTÍCULOS PENDIENTES DE APLICACIÓN SOCIOS COMERCIALES RELEVANTES PARA ESPAÑA

\begin{tabular}{|c|c|c|}
\hline Artíc. & Medida & F/ME \\
\hline \multicolumn{3}{|c|}{$\begin{array}{l}\text { Marruecos } \\
\text { Plazos pendientes de notificar } \\
\text { Pendiente de ratificación }\end{array}$} \\
\hline 5.1 & Normas para la eliminación de inspecciones reforzadas sobre alimentos, bebidas y piensos & $\mathrm{F}$ \\
\hline 7.1 & Inicio de la tramitación previa a la entrada para agilizar el levante & $\mathrm{F}$ \\
\hline 7.4 & Mantener sistemas de gestión de riesgo para control aduanero & ME \\
\hline 7.9 & Sistema de gestión especial para mercancías perecederas & $\mathrm{F}$ \\
\hline 10.4 & Ventanilla única aduanera & ME \\
\hline 10.5 & No realización de inspecciones previas en relación con la clasificación arancelaria y valoración en aduana & $\mathrm{F}$ \\
\hline \multicolumn{3}{|c|}{$\begin{array}{c}\text { Turquía } \\
\text { Plazos pendientes de notificar }\end{array}$} \\
\hline 7.9 & Sistema de gestión especial para mercancías perecederas & $\mathrm{F}$ \\
\hline \multicolumn{3}{|c|}{$\begin{array}{c}\text { China } \\
\text { Plazos pendientes de notificar }\end{array}$} \\
\hline 7.6 & Publicación de estadísticas de plazos de levante & ME \\
\hline 10.4 & Ventanilla única en un punto de entrada único & ME \\
\hline 10.9 & Sistema de tráfico de perfeccionamiento activo y pasivo & $\mathrm{F}$ \\
\hline 12 & Cooperación entre autoridades aduaneras & $\mathrm{F}$ \\
\hline \multicolumn{3}{|c|}{$\begin{array}{c}\text { Arabia Saudí } \\
\text { Plazos pendientes de notificar }\end{array}$} \\
\hline 2 & Procedimiento de consulta previa a la elaboración de normas & ME \\
\hline 10.4 & Ventanilla única aduanera & $\mathrm{ME}$ \\
\hline \multicolumn{3}{|c|}{$\begin{array}{c}\text { Brasil } \\
\text { Plazos notificados }\end{array}$} \\
\hline 7.1 & Inicio de la tramitación previa a la entrada para agilizar el levante. Se aplicará a partir del 31.12.17 & $\mathrm{F}$ \\
\hline 7.7.3 & $\begin{array}{l}\text { Puesta en práctica de un sistema ágil para operadores económicos autorizados. Número mínimo de medidas de } \\
\text { facilitación de comercio a aplicar a operadores económicos autorizados. En la práctica, al no asumir estas } \\
\text { obligaciones, cualquier otra disposición sobre OEA queda vacía de contenido. Se aplicará a partir de } 31.12 .17\end{array}$ & $\mathrm{~F}$ \\
\hline 11.9 & Inicio de la tramitación previa a la entrada para mercancías en tránsito. Se aplicará a partir del 31.12.19 & $\mathrm{F}$ \\
\hline \multicolumn{3}{|c|}{$\begin{array}{c}\text { India } \\
\text { Compromisos que entrarán en vigor el 22.2.2022 }\end{array}$} \\
\hline $1.1 .1 \mathrm{~b}$ & Publicación de los tipos arancelarios e impuestos aplicables & $\mathrm{F}$ \\
\hline 1.2.1 c & Facilitar los datos de contacto de los servicios de información aduaneros & $\mathrm{F}$ \\
\hline 1.3 & Disponer de punto(s) de información aduanara que responda(n) a peticiones de información de forma gratuita & ME \\
\hline 1.4 & Notificar a la OMC los puntos de información & $\mathrm{F}$ \\
\hline 2 & Procedimiento de consulta previa a la elaboración de normas & ME \\
\hline 3 & Emisión de resoluciones anticipadas & $\mathrm{F}$ \\
\hline 4 & $\begin{array}{l}\text { Procedimientos de recurso de decisiones aduaneras. China no asume apartados sobre plazos ni sobre motivación } \\
\text { de decisiones administrativas }\end{array}$ & \\
\hline 5.1.b & $\begin{array}{l}\text { Controles reforzados para alimentos, bebidas o piensos. China no asume el compromiso de uniformidad en la } \\
\text { aplicación de medidas sólo a puntos de entrada donde sean necesarias por condiciones sanitarias y fitosanitarias }\end{array}$ & \\
\hline 6.3.6 & Sanciones. Atenuante para reveladores de información & ME \\
\hline 7.1 .2 & Aplicación de procedimientos electrónicos para la tramitación previa a la entrada & ME \\
\hline 7.3 & Separación del levante y la determinación de los derechos de aduana & \\
\hline 7.4 & Mantener sistemas de gestión de riesgo para control aduanero & ME \\
\hline 7.8 .2 & Envíos urgentes. Reducción de documentación exigida & $\mathrm{F}$ \\
\hline 7.9 .4 & Mercancías perecederas. Comunicación de los motivos de demora & ME \\
\hline $8.2 \mathrm{a}$ & $\begin{array}{l}\text { Cooperación entre organismos que actúan en frontera (horarios, procedimientos, servicios comunes, controles } \\
\text { conjuntos, una sola parada) }\end{array}$ & ME \\
\hline 10.2 .2 & No obligación de presentar un documento original varias veces a diferentes autoridades & \\
\hline 10.4 & Ventanilla única aduanera & ME \\
\hline
\end{tabular}


TABLA 2 (continuación)

\begin{tabular}{|c|c|c|}
\hline 10.5 .2 & Inspección previa a la expedición. Compromiso de no introducir nuevas prescripciones & $\mathrm{ME}$ \\
\hline 10.9.2 & Sistema de tráfico de perfeccionamiento activo y pasivo & $\mathrm{F}$ \\
\hline 11.9 & Tránsito. Poder iniciar la tramitación previa a la entrada para mercancías en tránsito & $\mathrm{F}$ \\
\hline 11.10 & Tránsito. Dar por terminado el tránsito con la salida de la mercancía & $\mathrm{F}$ \\
\hline 11.13 & Tránsito. Posibilidad de ofrecer garantías globales para transacciones múltiples & $\mathrm{F}$ \\
\hline 11.15 & Tránsito. Limitación de uso de escoltas aduaneras a situaciones de alto riesgo & $\mathrm{F}$ \\
\hline 11.16 & $\begin{array}{l}\text { Tránsito. Necesidad de cooperar con otras autoridades aduaneras en materia de cargas, formalidades, funciona- } \\
\text { miento práctico }\end{array}$ & $\mathrm{ME}$ \\
\hline \multicolumn{3}{|c|}{$\begin{array}{l}\text { Chile } \\
\text { Plazos pendientes de notificar }\end{array}$} \\
\hline 7.7 & Establecimiento de régimen simplificado para operadores económicos autorizados & $\mathrm{F}$ \\
\hline \multicolumn{3}{|c|}{$\begin{array}{c}\text { Argentina } \\
\text { Compromisos que entrarán en vigor en } 2022 \\
\text { Pendiente de ratificación }\end{array}$} \\
\hline 10.2.1 & Aceptación de copias electrónicas & $\mathrm{ME}$ \\
\hline 10.4.1 & Ventanilla única aduanera & $\mathrm{ME}$ \\
\hline \multicolumn{3}{|c|}{$\begin{array}{l}\text { EAU } \\
\text { Plazos pendientes de notificar }\end{array}$} \\
\hline 3.1 & Emisión de resoluciones anticipadas & $\mathrm{ME}$ \\
\hline 7.1 & Inicio de la tramitación previa a la entrada para agilizar el levante & $\mathrm{F}$ \\
\hline 7.6 & Publicación de estadísticas de plazos de levante & $\mathrm{ME}$ \\
\hline 7.7 & Establecimiento del régimen simplificado para operadores económicos autorizados & $\mathrm{F}$ \\
\hline 10.4 & Ventanilla única aduanera & $F$ \\
\hline
\end{tabular}

\section{Conclusión}

El Acuerdo sobre la Facilitación del Comercio es muy innovador en muchos aspectos, puesto que mezcla elementos clásicos de regulación (compromisos firmes y disciplinas vinculantes) con otros elementos de mejores esfuerzos, además de ser muy flexible en cuanto a los plazos de aplicación.

Existe un consenso generalizado acerca del elevado beneficio potencial del acuerdo tanto por el lado de las exportaciones como por el de las importaciones. Sin embargo, el análisis de las notificaciones recibidas hasta el momento pone de manifiesto la existencia de diferentes grados de ambición y dificultades para la adopción de compromisos. Existen muchos países que han asumido todo o casi todo el AFC desde el momento de su entrada en vigor. En otros casos, no obstante, se observa que existen inercias administrativas que ralentizan la adecuada coordinación entre instituciones ligadas a la aduana. También se observa una clara dificultad para adoptar sistemas de ventanilla única y procedimientos telemáticos, así como para implantar la figura del operador económico autorizado, tres elementos que figuran entre los menos notificados. En varios de los destinos más importantes de nuestra exportación se reproduce este mismo análisis.

Conviene seguir realizando un estudio periódico de la evolución del AFC y de las necesidades técnicas para su adopción. La OMC está adoptando medidas pioneras a tal fin, como la creación de una base de datos de notificación de compromisos, la coordinación de programas de asistencia con numerosas instituciones (OMA, Banco Mundial, UNCTAD, Centro de Comercio Internacional, instituciones de integración regional...), etcétera. No son los recursos financieros los que resultan más escasos para tal fin, sino la capacidad de coordinar $D$ 
Representación Permanente de España ante los Organismos Internacionales en Ginebra

de forma efectiva los programas de ayuda técnica, la continuidad de las políticas y también la diferente percepción de la naturaleza winwin del acuerdo.

El éxito del AFC sólo se podrá evaluar en el medio y largo plazo, haciendo uso de indicadores objetivos del funcionamiento de las aduanas. En ese momento se podrá juzgar si las flexibilidades del AFC son un modelo adecuado para otras áreas de negociación. También en ese momento se verá si es necesario y posible reabrir el acuerdo para incorporar elementos que no fue posible introducir en la negociación.

ANEXO I

RATIFICACIONES Y NOTIFICACIONES DE LOS PRINCIPALES SOCIOS COMERCIALES

Principales socios comerciales de disposiciones del AFC con plazos ya definidos

\begin{tabular}{|c|c|c|c|}
\hline Miembro de la OMC & A & B & $\begin{array}{l}\text { Plazos todavía } \\
\text { no definidos * }\end{array}$ \\
\hline Angola * & & & 100,0 \\
\hline Arabia Saudí & 97,1 & & 2,9 \\
\hline Argentina * & 94,6 & 5,4 & \\
\hline Australia & 100,0 & & \\
\hline Bolivia * & & & 100,0 \\
\hline Brasil & 95,8 & 4,2 & 0,0 \\
\hline Chile & 92,9 & & 7,1 \\
\hline China & 75,4 & & 24,6 \\
\hline Colombia * & 96,7 & & 3,3 \\
\hline Corea & 100,0 & & \\
\hline Costa Rica * & 97,9 & & 2,1 \\
\hline Cuba * & & & 100,0 \\
\hline EAU & 89,2 & & 10,8 \\
\hline Ecuador * & 20,4 & & 79,6 \\
\hline EE UU & 100,0 & & \\
\hline Egipto * & 20,0 & & 80,0 \\
\hline El Salvador & 77,5 & & 22,5 \\
\hline Filipinas & 88,7 & & 11,3 \\
\hline Guatemala & 64,2 & & 35,8 \\
\hline Honduras & 58,7 & & 41,3 \\
\hline Hong Kong (China) & 100,0 & & \\
\hline India & 70,8 & 29,2 & \\
\hline Indonesia * & 5,4 & & 94,6 \\
\hline
\end{tabular}

\begin{tabular}{|c|c|c|c|}
\hline Islandia & 100,0 & & \\
\hline Israel * & 100,0 & & \\
\hline Japón & 100,0 & & \\
\hline Kazajstán & 42,5 & & 57,5 \\
\hline Kenia & 7,1 & & 92,9 \\
\hline Kuwait * & 59,6 & & 40,4 \\
\hline Macedonia & 97,5 & & 2,5 \\
\hline Malasia & 94,2 & & 5,8 \\
\hline Marruecos * & 91,2 & & 8,8 \\
\hline México & 100,0 & & \\
\hline Mongolia & 23,8 & & 76,2 \\
\hline Nueva Zelanda & 100,0 & & \\
\hline Nicaragua & 73,7 & & 26,3 \\
\hline Nigeria & 15,0 & & 85,0 \\
\hline Noruega & 100,0 & & \\
\hline Pakistán & 25,4 & & 74,6 \\
\hline Panamá & 74,6 & & 25,4 \\
\hline Paraguay & 52,5 & & 47,5 \\
\hline Perú & 61,2 & & 38,8 \\
\hline Qatar * & 92,9 & & 7,1 \\
\hline R. Dominicana & 72,5 & & 27,5 \\
\hline R.D. Congo * & 42,1 & & 57,9 \\
\hline Rusia & 100,0 & & \\
\hline Senegal & 45,0 & & 55,0 \\
\hline Singapur & 100,0 & & \\
\hline Sri Lanka & 25,8 & & 74,2 \\
\hline Sudáfrica * & & & 100,0 \\
\hline Suiza & 100,0 & & \\
\hline Tailandia & 91,7 & & 8,3 \\
\hline Taipei Chino & 100,0 & & \\
\hline Túnez * & 59,2 & & 40,8 \\
\hline Turquía & 97,9 & & 2,1 \\
\hline Ucrania & 31,3 & & 68,7 \\
\hline UE-28 & 100,0 & & \\
\hline Uruguay & 97,1 & 2,9 & \\
\hline Venezuela * & & & 100,0 \\
\hline Vietnam & 22,9 & & 77,1 \\
\hline \multicolumn{4}{|c|}{$\begin{array}{l}\text { A: ya entró en vigor, salvo que el país no haya ratificado } \\
\text { B: plazo ya conocido } \\
\text { Pte: Plazo todavía no conocido por falta de notificación o } \\
\text { ratificación } \\
\text { * Pendiente de ratificación: los compromisos y plazos que ha- } \\
\text { yan sido notificados no son todavía aplicables. } \\
\text { Fuente: elaboración propia a partir de la base de datos del } \\
\text { AFC. OMC. www.tfadatabase.org } \\
\text { Fecha de actualización: } 7 \text { de abril de } 2017 \text {. }\end{array}$} \\
\hline
\end{tabular}

